\title{
Análisis traductológico de culturemas en Cien años de soledad.
}

\section{Equivalencia y técnicas de traducción*}

Recepción: 12 de octubre de 2017

Forma de citar este artículo: Buitrago Cabrera, M. (2019). Análisis traductológico de culturemas en Cien años de soledad. Cuadernos De Lingüística Hispánica, (34), pp 67-85.

* Artículo de investigación producto del trabajo de grado realizado por la autora y dirigido por el Dr. Phil. Sergio Bolaños Cuéllar, Universidad Nacional de Colombia.

** Licenciada en Lenguas Extranjeras y magíster en Lingüística, Universidad Pedagógica y Tecnológica de Colombia. Actualmente cursa tercer semestre de Maestría en Lingüística Computacional en la Universidad de Stuttgart, Alemania, donde continúa investigando la traducción de especificidades culturales, ahora a partir de la inteligencia artificial. Correo electrónico: mariacamila.buitrago@uptc.edu.co iD https://orcid.org/0000-0002-2576-562X 


\title{
Resumen
}

Este artículo presenta un análisis de enfoque mixto acerca del tratamiento de culturemas en la traducción al inglés de la novela Cien años de soledad. Los culturemas, extraídos mediante un corpus doble, fueron clasificados en ámbitos culturales, también se identificaron las técnicas de traducción utilizadas para su recreación, y los tipos y rangos de equivalencia se determinaron a través del Modelo Traductológico Dinámico (MDT). El producto de esta investigación contribuye a la consolidación y aceptación del término culturema en el ámbito de la traductología y además demuestra que la integración de dos propuestas teóricas y metodológicas, como la traducción con orientación lingüístico-textual y la corriente cultural del funcionalismo, resulta útil para el desarrollo de un instrumento de análisis de culturemas en obras literarias.

Palabras clave: culturema, Cien años de soledad, traductología, cultura, equivalencia, técnicas de traducción.

\section{Traductological Analysis of Culturemes in One Hundred Years of Solitude.}

\section{Equivalence and Translation Techniques}

\begin{abstract}
This article presents a mixed focus analysis about the treatment of culturemes in the English translation of the novel One Hundred Years of Solitude. The culturemes, extracted by means of a double corpus, were classified in cultural fields, the translation techniques were also identified, and the types and ranges of equivalence were determined through the Dynamic Traductological Mode (MDT, by its acronym in Spanish). The product of this research contributes to the consolidation and acceptance of the term culture$m e$ in the traductology's topic and also demonstrates that the integration of two theoretical and methodological proposals, such as translation with linguistic-textual orientation and the cultural current of functionalism, is useful for the development of an instrument for analyzing culturemes in literary works.
\end{abstract}

Keywords: cultureme, One Hundred Years of Solitude, traductology, culture, equivalence, translation techniques. 


\section{Analyse en traductologie de culture-thèmes dans Cent ans de solitude. Équivalence et techniques de traduction}

\section{Résumé}

Cet article présente une analyse d'approche mixte par rapport au traitement de culture-thèmes dans la traduction en anglais du roman Cent ans de solitude. Les culturethèmes, tirés au moyen d'un corpus double, ont été classés en domaines culturels. On a aussi identifié les techniques de traduction utilisées pour sa reconstitution et les types de rangs d'équivalence ont été déterminés à travers le Modèle Traductologique Dynamique (MDT). Le produit de cette recherche contribue à consolider et accepter le terme culturème dans le domaine de la traductologie. En plus, cela démontre que l'intégration de deux propositions théoriques et méthodologiques, comme la traduction avec orientation linguistique-textuelle et le courant culturel du fonctionnalisme, devient utile pour le développement d'un instrument d'analyse de culturèmes dans des œuvres littéraires.

Palabras clave: culturème, Cent ans de solitude, traductologie, culture, équivalence, technique de traduction.

\section{Análise traductológica dos culturemas do livro "Cem anos de solidão". Equivalência e técnicas de tradução}

\section{Resumo}

Este artigo apresenta uma análise de foco misto sobre 0 tratamento de culturemas na tradução em inglês do romance Cem Anos de Solidão. Os culturemas, extraídos por meio de um corpus duplo, foram classificados em campos culturais, as técnicas de tradução utilizadas para recreação também foram identificadas e os tipos e faixas de equivalência foram determinados por meio do Modelo Dinâmico de Tradução (MDT). 0 produto desta pesquisa contribui para a consolidação e aceitação do termo culturema no campo da traductología e também demonstra que é útil a integração de duas propostas teóricas e metodológicas, como a tradução com orientação linguístico-textual e a corrente cultural do funcionalismo. É útil pelo desenvolvimento de um instrumento para analisar culturemas em obras literárias.

Palavras-chave: culturema, Cem Anos de Solidão, traductología, cultura, técnicas de tradução de equivalência. 


\section{Introducción}

¿Por qué nombramos el mundo de formas tan distintas? ¿De qué forma un sistema cultural particular se ve reflejado en las representaciones lingüísticas de su comunidad? En esta investigación nos centramos en el estudio de una situación comunicativa, intercultural e interlingüística muy desafiante: la traducción de "culturemas".

Los culturemas, entendidos como fenómenos socioculturales que se manifiestan lingüística y extralingüísticamente, representan todo un desafío para los traductores que frecuentemente enfrentan la inexistencia de un concepto marcado culturalmente en una cultura meta mientras que en la cultura origen el mismo concepto es parte del imaginario común. Se entiende entonces que los culturemas están inscritos en la mentalidad, costumbres, tradiciones, condiciones climáticas, gastronomía, humor, gestualidad, entre muchos otros constituyentes identitarios de un pueblo.

En este trabajo nos propusimos identificar, clasificar por ámbitos culturales, y analizar la recreación en inglés de los culturemas presentes en la novela Cien años de soledad para, posteriormente, describir las técnicas de traducción utilizadas y su tipo de equivalencia. Esto, a través de la integración de dos propuestas teóricas y metodológicas, por una parte, el Modelo Traductológico Dinámico de Bolaños (2008), desde la traducción con orientación lingüístico-textual, y por otra, el enfoque dinámico y funcional de las técnicas de traducción de Molina (2001), desde la corriente cultural del funcionalismo.

El producto de esa investigación contribuye en lo teórico a la consolidación y aceptación del término "culturema" en el ámbito de la traductología, y en lo práctico ofrece un instrumento para el análisis de traducción literaria u otros tipos de obras con alta carga cultural.

En el primer apartado presentamos las perspectivas lingüística y cultural de la traducción a fin de contextualizar al lector con teorías que tienen su origen en la relación cultura-lenguaje/cultura-traducción hasta llegar al culturema, elemento en el cual convergen. En seguida exponemos la metodología, en la cual se plantea e implementa la propuesta integradora. Finalizamos con los resultados, la discusión sobre estos y las conclusiones obtenidas después de analizar la utilidad del instrumento diseñado.

\section{Fundamentación teórica}

A continuación analizamos algunos postulados sobre la traducción con orientación lingüística y cultural. Ambas perspectivas han demostrado ser complementarias, 
pues su principal elemento común es el reconocimiento de la traducción como un proceso comunicativo.

\subsection{Orientación lingüística}

Esta corriente traductológica de orientación predominantemente lingüística, de acuerdo con Bolaños (2016b), "parte del texto hacia el contexto e intenta comprender la naturaleza textual del proceso y, en segunda instancia, se preocupa por el contexto sociocultural de la producción de la traducción” (p. 3).

Según Levý (1967), traductólogo checo de la corriente literaria (citado por Bolaños, 2016b, p. 14), la traducción se aborda mediante el principio "minimax": mínimas pérdidas, máximas ganancias. Su definición de traducción coincide con los intereses de esta investigación:

Proceso comunicativo unidireccional, intralingüístico e intercultural, de dos fases, en el cual, a partir del análisis traductológico del texto primario se crea un texto secundario (metatexto), que sustituye al primario en otro ámbito lingüístico-cultural.

Proceso que se caracteriza por la orientación hacia la transmisión del efecto comunicativo del texto primario, con modificaciones parciales de acuerdo con las diferencias entre las dos lenguas, las dos culturas y las dos situaciones comunicativas. (Levý, 1967, citado por Bolaños, 2016b, p. 14)

\subsection{Orientación cultural}

" $[\mathrm{L}] \mathrm{a}$ traductología orientada hacia los estudios literarios, culturales y filosóficos parte del contexto, así como del papel que desempeña el traductor y de allí, en algunas oportunidades, pasa al texto", afirma (Bolaños, 2016b, p. 3), y agrega que su interés fundamental se centra tanto en la actividad del traductor, como en la comprensión de la naturaleza sociocultural, histórica y política de la traducción para, en segundo plano, ocuparse de la relación texto origen-texto meta.

En cuanto a los cambios de perspectiva que permitieron a los estudios de traducción considerar la cultura en la que se inscriben los textos, Carbonell (1999, p. 25) destaca como uno de los más relevantes el enfoque cultural adoptado por Albrecht Neubert y Mary Snell-Hornby (1988) con el denominado "análisis de arriba hacia abajo" (topdown), que consiste en partir de las categorías más amplias y complejas (el macronivel del texto situado en su contexto cultural) e ir descendiendo hasta las categorías más sim- 
ples (el micronivel de la palabra y de la frase). Este cambio de paradigma ha sido llamado "El giro cultural" o the cultural turn in translation studies.

\subsection{Cultura y lenguaje}

Para la construcción de este concepto consideramos la versión menos radical de la hipótesis Sapir-Whorf, en la cual el lenguaje no determina completamente, pero sí influye en el pensamiento y la percepción, así como los aportes de Hatim y Manson (1995), según los cuales las lenguas difieren en el modo de percibir el mundo y, en consecuencia, compartimentan la realidad. Este postulado es explicado desde la semántica contrastiva en la obra de Nida y Taber (1974), donde los autores afirman que las lenguas difieren sobre todo en las clasificaciones semánticas más generales, es decir, en la manera más general de organizar la realidad. Esto nos lleva a comprender que la traducción es más que una tarea lingüística, porque abarca además la dimensión cultural determinada por la percepción.

Con el propósito de operacionalizar los conceptos de cultura y lenguaje, consideramos las posturas de Goodenough (1964) y de Komissarov (2002). El primero, (citado por Hennecke, 2015), plantea que la cultura no existe independientemente del sujeto:

[...] a society's culture consists of whatever it is one has to know or believe in order to operate in a manner acceptable to its members, [...] it's not a material phenomenon; it does not consist of things, people, behaviour, or emotions. It is rather an organization of these things. It is the form of things that people have in mind, their models of perceiving, recording, and otherwise interpreting them ${ }^{1}$. (p. 110)

Sobre el segundo aspecto, Komissarov (citado por Bolaños, 2016b) considera que "el lenguaje como instrumento de comunicación verbal es una parte importante de la cultura y todas sus particularidades estructurales y funcionales se pueden considerar manifestaciones de la cultura del colectivo lingüístico correspondiente" (p. 33). En sintonía con Komissarov, Agar (2006, p. 6) afirma que "la cultura es una construcción artificial e intersubjetiva que permite la traducción entre unos y otros"2.

1 [... la cultura de una sociedad consiste en todo aquello que uno tiene que saber o creer para operar de manera aceptable como miembro, [...] no es un fenómeno material; no consta de cosas, personas, comportamiento o emociones. Es más bien una organización de dichas cosas. Es la configuración mental que las personas tienen de esas cosas, los modelos que usan para percibirlas, memorizarlas y demás formas para interpretarlas. (Traducción libre de la autora).

2 Todas las citas con referencia a Agar (2006) son traducción directa y libre de la autora. 
Teniendo en cuenta las características y propósitos comunicativos del lenguaje, esta investigación concibe la relación cultura-lenguaje como una simbiosis donde la lengua representa en gran medida el sistema cultural de un pueblo, pero no condiciona sus características, ni viceversa. Adicionalmente, nos adherimos a la visión plural y contrastiva de cultura que plantea Agar (2006) y definimos la traducción como el ente mediador que permite la decodificación, la comprensión y la interacción entre dos lenguas-culturas.

\subsection{La cultura en la traducción}

La interacción lenguaje-estructura social y lenguaje-cultura plantea a menudo conflictos sociolingüísticos en la traducción. Bolaños (2016b, p. 72) explica a partir de Shveitser (1988) que la traducción como reflejo del mundo social revela dos aspectos: el vínculo directo de aquello que se transmite en el texto traducido con el sistema sociocultural del original y la relación del texto traducido con el reflejo mediado de la diferenciación social. Visto así, el problema de la traducción de un concepto, vinculado a una realidad específica, tiene que ver con la búsqueda de análogos sociales en la cultura meta.

Tal búsqueda parte de un problema estructural, la falta de consenso ante la noción misma de cultura. En un intento por llegar a una definición satisfactoria, que se ajustara a las necesidades de este estudio, nos apegamos a la visión antropológica de la percepción cultural de Agar (2006), en la cual la cultura no existe per se sino que surge de la incomprensión ante lo ajeno (p.5).

De esta forma, comprendemos la cultura como una construcción producto de la interacción entre la lengua-cultura 1, en este caso el texto origen (T0) y la lengua-cultura 2 o texto meta (TM). Ubicar la cultura justo en medio de las situaciones conflictivas es, para este caso, la forma más efectiva de comprender los fenómenos culturales en una obra que ha sobrepasado sus propios límites geográficos.

\subsection{El culturema}

La traducción que construimos es la cultura que describimos. (Agar, 2006)

La noción de culturema nació entre los teóricos funcionalistas, dentro de los cuales Bolaños (2016b) destaca a Oksaar (1988), Vermeer (1983), y Nord (1994, 1997). Este último solía referirse a ellos como indicadores culturales o puntos ricos de diferencia entre dos culturas. Kade (1968) también sobresale en esta lista por denominar como realia a todo fenómeno e institución socioeconómica y culturalmente determinada. 
Mientras que Catford (1970) hablaba de un problema de intraducibilidad relacionado con la inexistencia de un sustituto léxico o sintáctico en la lengua meta (p.157).

Por su parte, Hennecke (2015) concluye que las diferencias culturales se hacen notorias cuando, en el ejercicio de la traducción, el significado de una expresión, de una frase 0 de un texto completo resulta difuso, cuestionable o completamente incomprensible (p. 115).

Molina (2001) retoma a Nord (1997) para definir el culturema como

[...] un elemento verbal o paraverbal que posee una carga cultural específica en una cultura y que al entrar en contacto con otra cultura a través de la traducción puede provocar un problema de índole cultural entre los textos origen y meta (p. 89).

La misma autora plantea la dimensión dinámica del culturema por medio de tres características: 1) los culturemas no existen en el marco de una única cultura, sino como consecuencia de un trasvase cultural, 2) los culturemas pueden surgir en el marco de dos culturas concretas sin ser por esto universales, y 3) la función del culturema depende del contexto en el que aparezca.

\section{Metodología}

Esta investigación presenta un enfoque mixto y un carácter descriptivo a través de los cuales determinamos, por una parte, las técnicas de traducción empleadas y, por otra, establecemos el tipo de equivalencia alcanzada.

Para este propósito trabajamos con un corpus doble ${ }^{3}$ de culturemas. En primera instancia, llevamos a cabo la extracción manual de construcciones con carga cultural que pudieran ser clasificadas dentro del instrumento de ámbitos culturales (medio natural, patrimonio cultural, cultura social, y cultura lingüística). Posteriormente, rastreamos las recreaciones de estas expresiones en la novela traducida con ayuda del enfoque dinámico y funcional de las técnicas de traducción, ambos instrumentos propuestos por Molina (2001). El método de análisis para determinar los tipos de equivalencia fue el Modelo Traductológico Dinámico (MTD) de Bolaños (2008), en el cual prima la dimensión pragmática de la equivalencia y la fidelidad a la intención original del autor.

3 El corpus inicial fue posteriormente validado y filtrado por grado de idiomaticidad, extrañeza o ambigüedad en la forma de la recreación en inglés. 


\subsection{Propuesta integradora para el análisis del culturema}

Esta propuesta integra el concepto de equivalencia traductora, que construimos a partir de la revisión de los planteamientos surgidos durante los últimos 40 años; el Modelo Traductológico Dinámico, que ayuda a explicar la naturaleza de la equivalencia traductora, y finalmente las técnicas de traducción a partir de un enfoque dinámico y funcional, capaz de apoyar el análisis y la catalogación del funcionamiento de equivalencia.

2.1.1 La equivalencia traductora. Shveitser (1988) revisa la propuesta de Nida y Taber (1969) sobre la equivalencia dinámica, que se define como la característica de la traducción, según la cual "el contenido semántico del original se transmite en la lengua del receptor, de modo que la respuesta del receptor de la traducción sea semejante a la reacción de los receptores del original" (Bolaños, 2016b, p.15). El mismo autor agrega que, para Shveitser, la exigencia de la equivalencia comunicativa pragmática es la más importante, por cuanto esto implica la transmisión del efecto comunicativo del texto origen, así como para Komissarov (citado por Bolaños, 2016b) la recepción equivalente del contenido del original asegura la transmisión del potencial pragmático por medio de la traducción. No obstante, advierte que el colectivo lingüístico y la cultura del receptor de la traducción frecuentemente dificultan el logro de la equivalencia pragmática y es por esto que el traductor hace uso de diversos "ajustes" en el texto meta. Estos ajustes se ven representados en las técnicas de traducción que analizamos más adelante.

2.1.2 El Modelo Traductológico Dinámico (MDT). Bolaños (2016a) presenta una aproximación moderna de la equivalencia traductora que gira en torno a la relación existente entre la posición de equivalencia por defecto (PED), el rango de equivalencia (RE), las instrucciones del iniciador (II) y las normas de traducción (NT). Este modelo resulta adecuado para dar cuenta de los diferentes tipos de equivalencia que se presentan entre diversos tipos de texto: literario, científico, y apelativo al momento de emplear las diferentes estrategias de traducción.

El MDT ubica a la pragmática como la dimensión guía en la construcción del texto traducido. Por lo tanto, se trata de un modelo comunicativo que incluye a los participantes del proceso traductor, explica la naturaleza de la equivalencia y describe los factores claves del proceso traductor.

La posición de equivalencia por defecto (PED) es la relación que se establece entre el texto de la lengua origen y el texto de la lengua meta, en la cual prevalece la intención comunicativa del autor original. En cuanto al rango de equivalencia (RE), se trata de un constructo teórico útil para comprender que al aplicar la PED se activa un rango de 
otros posibles equivalentes determinados por las decisiones del traductor, las normas de traducción o las instrucciones del iniciador.

La posición de equivalencia por defecto (PED) y el rango de equivalencia (RE) son los dos componentes del modelo que se han tenido en cuenta para esta investigación, por cuanto el primero permite verificar el tipo de equivalencia en la traducción de los culturemas, y el segundo permite analizar las distintas posibilidades para recrearlos siguiendo, primordialmente, la dimensión pragmática de la equivalencia y siendo fiel a la intención original del autor.

2.1.3 Las técnicas de traducción, un enfoque dinámico y funcional. Molina y Hurtado (2002) consideran que la definición de una técnica traductora y la apreciación de su validez se deben considerar por separado para preservar la dimensión dinámica de la traducción. Desde esta perspectiva, el método opera en función del objetivo mismo de la traducción, tiene carácter supraindividual y recorre todo el texto. La estrategia es el mecanismo que el traductor utiliza para resolver problemas y es de carácter individual, mientras que la técnica es la aplicación concreta visible en el resultado y afecta a zonas menores del texto.

En este enfoque, Molina (2001) pone en consideración la reflexión de Hurtado Albir (1996) para proponer su propia definición de técnica de traducción: "procedimiento de análisis y catalogación del funcionamiento de la equivalencia traductora" (p.115) y les atribuye cinco características básicas 1 . Afectan al resultado de la traducción, 2. Se catalogan en comparación con el original, 3. Se refieren a microunidades textuales, 4. Tienen un carácter discursivo y contextual, 5. Son funcionales. Su propuesta se concreta en la clasificación de dieciocho técnicas de traducción con enfoque dinámico y funcional:

Tabla 1. Técnicas de traducción recopiladas por Molina (2001)

\begin{tabular}{|cl|c|}
\hline 1. & Adaptación & Reemplazar un elemento cultural por otro propio de la cultura receptora. \\
\hline 2. & Ampliación lingüística & $\begin{array}{c}\text { Añadir elementos lingüísticos. Recurso especialmente utilizado en interpretación } \\
\text { consecutiva y doblaje. }\end{array}$ \\
\hline 3. & Amplificación & $\begin{array}{c}\text { Introducir precisiones no formuladas en el texto original: informaciones, paráfrasis } \\
\text { explicativas, notas de pie de página. }\end{array}$ \\
\hline 4. & Calco & $\begin{array}{c}\text { Traducir literalmente una palabra o un sintagma extranjero; puede ser léxico y es- } \\
\text { tructural. }\end{array}$ \\
\hline 5. & Compensación & $\begin{array}{c}\text { Introducir en otro lugar del texto meta un elemento de información o efecto estilístico } \\
\text { que no se ha podido reflejar en el mismo lugar en que aparece situado en el texto original. }\end{array}$ \\
\hline 6. & $\begin{array}{c}\text { Comprensión lin- } \\
\text { güística }\end{array}$ & $\begin{array}{c}\text { Sintetizar elementos lingüísticos. Recurso especialmente utilizado en interpretación } \\
\text { simultánea y subtitulación. }\end{array}$ \\
\hline 7. & Creación discursiva & Establecer una equivalencia efímera, totalmente imprevisible fuera de contexto. \\
\hline
\end{tabular}




\begin{tabular}{|c|c|c|}
\hline 8. & Descripción & Reemplazar un término o expresión por la descripción de su forma y/o función. \\
\hline 9. & Equivalente acuñado & Utilizar un término o expresión reconocido como equivalente en la lengua meta. \\
\hline 10. & Generalización & Utilizar un término más general o neutro. \\
\hline 11. & Modulación & $\begin{array}{l}\text { Efectuar un cambio de punto de vista, de enfoque o de categoría de pensamiento en } \\
\text { relación con la formulación del texto original; puede ser léxica y estructural. }\end{array}$ \\
\hline 12. & Particularización & Utilizar un término más preciso o concreto. Se opone a la generalización. \\
\hline 13. & Préstamo & $\begin{array}{l}\text { Integrar una palabra o expresión de otra lengua tal cual. Puede ser puro (sin ningún } \\
\text { cambio) o naturalizado (normalizado a la grafía de la lengua meta). }\end{array}$ \\
\hline 14. & Reducción & $\begin{array}{l}\text { Suprimir en el texto meta algún elemento de información presente en el texto origi- } \\
\text { nal. Puede ser total o parcial. }\end{array}$ \\
\hline 15. & $\begin{array}{l}\text { Substitución (lingüís- } \\
\text { tica, paralingüística) }\end{array}$ & Cambiar elementos lingüísticos por paralingüísticos o viceversa. \\
\hline 16. & Traducción literal & Traducir palabra por palabra un sintagma o expresión, pero no una sola palabra. \\
\hline 17. & Transposición & Cambiar la categoría gramatical. \\
\hline 18. & Variación & $\begin{array}{l}\text { Cambiar elementos lingüísticos o paralingüísticos (entonación, gestos) que afectan } \\
\text { a aspectos de la variación lingüística: cambios de tono textual, estilo, dialecto social, dialecto } \\
\text { geográfico, etc. }\end{array}$ \\
\hline
\end{tabular}

Esta es la clasificación de técnicas que utilizamos para el análisis de los culturemas traducidos al inglés. En algunos casos, más de una técnica sobresale en la recreación de un culturema.

\subsection{Aplicación de la propuesta integradora}

Una matriz de análisis fue diseñada para operacionalizar la teoría y unificar los conceptos en torno al culturema en el texto origen (T0) y en el texto meta (TM). En la casilla CULTUREMA TO presentamos el fragmento original que constituye el culturema, en frente se indican las categorías gramaticales de la expresión. En la casilla CULTUREMA TM damos a conocer el fragmento traducido y en la segunda sección de la columna derecha, la técnica de traducción identificada.

En las dos últimas casillas incluimos dos de los cuatro aspectos del Modelo Traductológico Dinámico (MDT): la posición de equivalencia por defecto (PED), a partir de la cual indicamos el tipo de equivalencia alcanzada en la traducción y ofrecemos una breve descripción tanto de la expresión original como del proceso de recreación. El segundo aspecto corresponde al rango de equivalencia (RE), presente solo en algunos casos. Allí proponemos otras alternativas de traducción para el culturema bajo el parámetro de guardar fidelidad al efecto del original; esta sección fue incluida con la intención de demostrar el carácter sincrónico/diacrónico de la traducción, es decir, que no existe una única posibilidad de traducción, sino un rango amplio para otras recreaciones. 


\section{A continuación presentamos una muestra de los culturemas analizados por ámbi-} tos culturales en el formato original de la matriz propuesta.

\section{Cultura lingüística}

\begin{tabular}{|c|c|c|}
\hline $\mathrm{P}$ & CULTUREMA TO & CATEGORIA GRAMATICAL \\
\hline 239 & $\begin{array}{l}\text {-En vez de andar pensando en tus alocadas novelerías, debes ocuparte de tus hijos - } \\
\text { replicó-. Míralos cómo están, abandonados a la buena de Dios, igual que los burros. } \\
\text { (...), la abandonaron a la buena de Dios. Remedios, la bella, se quedó vagando por el } \\
\text { desierto de la soledad, (...) }\end{array}$ & $\begin{array}{l}\text { Expresión idiomática con } \\
\text { adjetivo y sustantivo }\end{array}$ \\
\hline P. & CULTUREMA TM & TÉCNICA DE TRADUCCIÓN \\
\hline 254 & $\begin{array}{l}\text { "Instead of going around thinking about your crazy inventions, you should be worrying } \\
\text { about your sons," she replied. "Look at the state they're in, running wild just like } \\
\text { donkeys." } \\
\text { (...), they let her go her own way. Remedios the beauty stayed there wandering through } \\
\text { the desert of solitude, (...) }\end{array}$ & Adaptación con reducción \\
\hline \multicolumn{3}{|c|}{$\begin{array}{l}\text { Posición de Equivalencia por Defecto (PED): Total } \\
\text { La adaptación de 'abandonar a la buena de Dios' por 'running wild'y 'let her go her own way' permite al lector comprender el } \\
\text { sentido de desamparo y desinterés sobre estas personas. En la reducción de 'a la buena de Dios' se pierde la carga religiosa de la } \\
\text { expresión. }\end{array}$} \\
\hline & ), they let her abandoned to God. Remedios t & \\
\hline
\end{tabular}

\begin{tabular}{|c|c|c|}
\hline P. & CULTUREMA TO & CATEGORÍA GRAMATICAL \\
\hline 29 & $\begin{array}{l}\text {..., el domingo trágico en que José Arcadio Buendía le ganó una pelea de gallos a } \\
\text { Prudencio Aguilar. Furioso, exaltado por la sangre de su animal,... -Te felicito -gritó-. } \\
\text { A ver si por fin ese gallo le hace el favor a tu mujer. }\end{array}$ & $\begin{array}{l}\text { Expresión fija de preposición e } \\
\text { infinitive } \\
\text { Expresión idiomática con verbo y } \\
\text { sustantivos. }\end{array}$ \\
\hline P. & CULTUREMA TM & TÉCNICA DE TRADUCCIÓN \\
\hline 23 & $\begin{array}{l}\text {...that tragic Sunday when José Arcadio Buendía won a cockfight from Prudencio } \\
\text { Aguilar. Furious, aroused by the blood of his bird,... "Congratulations!" he shouted. } \\
\text { "Maybe that rooster of yours can do your wife a favor." }\end{array}$ & $\begin{array}{l}\text { Compresión lingüística y equivalente } \\
\text { acuñado }\end{array}$ \\
\hline \multicolumn{3}{|c|}{$\begin{array}{l}\text { Posición de Equivalencia por Defecto (PED): Total } \\
\text { La expresión fija 'A ver si...' que en general expresa expectación o interés por saber algo es comprendida en el adverbio Maybe y es } \\
\text { curioso que el artículo definido en 'hacer el favor', que en español es fundamental para comprender la intención de la expresión, es } \\
\text { convertido en un artículo indefinido en inglés 'do your wife a favor'. Sin embargo, en la expresión recreada, y gracias al contexto } \\
\text { previo, queda clara la carga sexual del comentario. }\end{array}$} \\
\hline \multicolumn{3}{|c|}{$\begin{array}{l}\text { Rango de Equivalencia (RE): } \\
\text { "Congratulations!" he shouted. "Maybe that rooster of yours can do something for your wife." } \\
\text { "Congratulations!" he shouted. "Maybe that rooster of yours can help your wife." } \\
\text { "Congratulations!" he shouted. "Maybe that rooster of yours can do the deed to your wife." }\end{array}$} \\
\hline
\end{tabular}

\begin{tabular}{|l|c|c|}
\hline P. & \multicolumn{1}{|c|}{ CULTUREMA TO } & CATEGORÍA GRAMATICAL \\
\hline 111 & "Atrévete, asesino", gritaba. "Y mátame también a mí, hijo de mala madre". & $\begin{array}{l}\text { Sustantivos y adjetivo en } \\
\text { expresión diomática }\end{array}$ \\
\hline P. & \multicolumn{1}{|c|}{ CULTUREMA TM } & TÉCNICA DE TRADUCCIÓN \\
\hline 115 & "I dare you to, murderer! "she shouted. "And kill me too, son of an evil mother." & Traducción literal \\
\hline $\begin{array}{l}\text { Posición de Equivalencia por Defecto (PED): Parcial } \\
\text { La expresión 'hijo de mala madre' hace referencia a una mujer indecente o de mala reputación y no necesariamente, a una malvada } \\
\text { como se recrea con la traducción literal en el TM generando una posición de equivalencia parcial. }\end{array}$ \\
\hline
\end{tabular}




\begin{tabular}{|l|l|c|}
\hline P. & \multicolumn{1}{|c|}{ CULTUREMA TO } & CATEGORÍA GRAMATICAL \\
\hline 63 & $\begin{array}{l}\text { Y para que lo sepa de una vez, no necesitamos ningún corregidor porque aquí } \\
\text { no hay nada que corregir. }\end{array}$ & Sustantivo y verbo - juego de palabras. \\
\hline P. & \multicolumn{1}{|c|}{ CULTUREMA TM } & TÉCNICA DE TRADUCCIÓN \\
\hline 61 & $\begin{array}{l}\text { "And so that you know it once and for all, we don't need any judges here } \\
\text { because there's nothing that need judging." }\end{array}$ & Adaptación y variación \\
\hline $\begin{array}{l}\text { Posición de Equivalencia por Defecto (PED): Total } \\
\text { Este culturema surge por el juego de palabras que utiliza José Arcadio Buendía para rechazar la presencia del corregidor en } \\
\text { Macondo, se observa claramente el esfuerzo del traductor por mantener el efecto estilístico y para ese propósito adapta 'corregidor' } \\
\text { como 'judges' (jueces) porque de esta manera puede utilizar el sustantivo 'judging' (juicio) para expresar que no hay nada que } \\
\text { juzgar, así como en español no hay nada que corregir. El juego de palabras no se podría mantener si se sigue utilizando 'magistrate' } \\
\text { como inicialmente se tradujo este cargo. }\end{array}$
\end{tabular}

\begin{tabular}{|l|l|c|}
\hline P. & \multicolumn{1}{|c|}{ CULTUREMA TO } & CATEGORIA GRAMATICAL \\
\hline 105 & $\begin{array}{l}\text { Sin embargo, el día en que el médico le reveló el secreto a Aureliano, este le } \\
\text { sacó el cuerpo a la conspiración. }\end{array}$ & $\begin{array}{l}\text { Verbo con sustantivo y artículos en } \\
\text { expresión idiomática. }\end{array}$ \\
\hline $\mathbf{1}$ & \multicolumn{1}{|c|}{ CULTUREMA TM } & TÉCNICA DE TRADUCCIÓN \\
\hline 108 & $\begin{array}{l}\text { Nevertheless, the day the doctor revealed the secret to Aureliano, the latter } \\
\text { elicited the whole plan of the conspiracy. }\end{array}$ & Creación discursiva con modulación \\
\hline $\begin{array}{l}\text { Posición de Equivalencia por Defecto (PED): Nula } \\
\text { Este caso constituye un error de traducción, y por lo tanto no se puede establecer la intención de la técnica (sin embargo, el } \\
\text { resultado tiene rasgos de creación discursiva y modulación). El lapsus fue causado seguramente por interpretación literal de la } \\
\text { expresión 'sacar el cuerpo', que en la cultura origen designa la acción de evadir una acción. Quizás el traductor interpretó el } \\
\text { verbo sacar con el mismo sentido de la expresión 'sacar la cara por algo' y por eso lo recreó como 'elicit' que en realidad } \\
\text { significa provocar, promover, etc., y que para el caso describe una acción totalmente opuesta a la planteada en el TO y por lo } \\
\text { tanto no se establece ningún tipo de equivalencia. }\end{array}$ \\
$\begin{array}{l}\text { Rango de Equivalencia (RE): } \\
\text { - Nevertheless, the day the doctor revealed the secret to Aureliano, the latter brushed off the whole plan of the conspiracy. } \\
\text { - Nevertheless, the day the doctor revealed the secret to Aureliano, the latter backed down from the whole plan of the } \\
\text { conspiracy. }\end{array}$ \\
\hline
\end{tabular}

\begin{tabular}{|l|l|c|}
\hline P. & \multicolumn{1}{|c|}{ CULTUREMA TO } & CATEGORÍA GRAMATICAL \\
\hline 33 & $\begin{array}{l}\text { Pero José Arcadio la siguió buscando toda la noche en el olor de humo que ella } \\
\text { tenía en las axilas y que se le quedó metido debajo del pellejo. (...) }\end{array}$ & Preposición con sustantivo \\
\hline $\mathbf{P .}$ & \multicolumn{1}{|c|}{ CULTUREMA TM } & TÉCNICA DE TRADUCCIÓN \\
\hline $\mathbf{2 8}$ & $\begin{array}{l}\text { But José Arcadio kept looking for her all night long, for the smell of smoke that she } \\
\text { had under her armpits and that had got caught under his skin. }\end{array}$ & Particularización y Modulación \\
\hline $\begin{array}{l}\text { Posición de Equivalencia por Defecto (PED): Parcial } \\
\text { El pellejo es la piel de un animal, en especial cuando está separada del cuerpo. La elección de esta palabra en el TO tiene que } \\
\text { ver tal vez con la intención de describir una piel tenaz y maltratada por los años. La particularización 'skin' elimina esta } \\
\text { connotación y por lo tanto conduce a una modulación. Aunque el equivalente acuñado para pellejo en inglés es 'hide', } \\
\text { resultaría ser una construcción inusual. }\end{array}$ \\
\hline
\end{tabular}

\section{Cultura social}

\begin{tabular}{|l|l|c|}
\hline P. & \multicolumn{1}{|c|}{ CULTUREMA TO } & CATEGORÍA GRAMATICAL \\
\hline 282 & Úrsula no volvió a levantarse después de las nueve noches de Amaranta. & Sustantivos \\
\hline P. & \multicolumn{1}{|c|}{ CULTUREMA TM } & TÉCNICA DE TRADUCCIÓN \\
\hline 303 & Úrsula did not get up again after the nine nights of mourning for Amaranta \\
\hline $\begin{array}{l}\text { Posición de Equivalencia por Defecto (PED): Total } \\
\text { Las nueve noches de Amaranta en el TO se refieren al novenario de difuntos que en la tradición católica es una de las formas más } \\
\text { completas y eficaces de pedir por el eterno descanso de un creyente, se lleva a cabo después del sepelio y consiste en el rezo del } \\
\text { Santo Rosario durante nueve días. Con la amplificación 'mourning'que significa duelo, el lector meta puede inferir que se trata de } \\
\text { algún tipo de ritual póstumo. }\end{array}$ \\
\hline \multicolumn{2}{|l}{} \\
\hline \multicolumn{2}{|l|}{ Rango de Equivalencia (RE): Úrsula did not get up again after the nine nights of mourning and praying for Amaranta } \\
\hline
\end{tabular}




\begin{tabular}{|c|c|c|}
\hline P. & CULTUREMA TO & CATEGORÍA GRAMATICAL \\
\hline 50 & $\begin{array}{l}\text { Ponía jugo de naranja con ruibarbo en una cazuela que dejaba al sereno toda la } \\
\text { noche, y le daba la pócima al siguiente día en ayunas. }\end{array}$ & $\begin{array}{l}\text { Verbo con preposición contráctil y } \\
\text { sustantivo }\end{array}$ \\
\hline 338 & $\begin{array}{l}\text { Allí lo encontró Úrsula una tarde en que andaba asperjando la casa con agua } \\
\text { serenada y un ramo de ortigas, (...) }\end{array}$ & Sustantivo con adjetivo \\
\hline P. & CULTUREMA TM & TÉCNICA DE TRADUCCIÓN \\
\hline $46-47$ & $\begin{array}{l}\text { She put some orange juice and rhubarb into a pan that she left in the dew all night } \\
\text { and she gave her the dose the following day on an empty stomach. } \\
\text { Úrsula found him there one afternoon when she was going about sprinkling the } \\
\text { house with distilled water and a bunch of nettles, (...) }\end{array}$ & Equivalente acuñado \\
\hline \multicolumn{3}{|c|}{$\begin{array}{l}\text { Posición de Equivalencia por Defecto (PED): Parcial } \\
\text { La expresión 'dejar al sereno' pertenece por completo a la cultura origen donde el sereno es más que la humedad que impregna la } \\
\text { atmósfera durante la noche. En este fragmento se le atribuyen propiedades curativas que al parecer pueden intensificar las } \\
\text { cualidades del jugo de naranja y del rubiarbo. El equivalente acuñado 'the dew' se refiere más exactamente al rocío de la mañana, } \\
\text { sin embargo, es posible que el lector, que no conoce las prácticas populares de la lengua origen, pueda percibir esta práctica como } \\
\text { un exotismo propio del Caribe colombiano. } \\
\text { En el segundo caso se observa la particularización 'distilled' utilizada para el adjetivo 'serenada'. El agua serenada no tiene el } \\
\text { proceso de purificación del agua destilada, pero da la sensación de cumplir con el propósito del TO. }\end{array}$} \\
\hline \multicolumn{3}{|c|}{$\begin{array}{l}\text { Rango de Equivalencia (RE): } \\
\text { - She put some orange juice and rhubarb into a pan that she left out in the open all night... } \\
\text { - She put some orange juice and rhubarb into a pan that she left in the night dew all night... }\end{array}$} \\
\hline
\end{tabular}

\begin{tabular}{|l|l|c|}
\hline P. & \multicolumn{1}{|c|}{ CULTUREMA TO } & CATEGORIA GRAMATICAL \\
\hline 336 & $\begin{array}{l}\text { (...) en cierta ocasión se resignaron a comer mazamorra por tres días para que ella } \\
\text { pudiera compara un mantel holandés. }\end{array}$ & Sustantivo \\
\hline P. & \multicolumn{1}{|c|}{ CULTUREMA TM } & TÉCNICA DE TRADUCCIÓN \\
\hline 364 & $\begin{array}{l}\text { (...) on a certain occasion they resigned themselves to eating crumbs for three days so } \\
\text { that she could buy a Dutch tablecloth. }\end{array}$ & Generalización con modulación \\
\hline $\begin{array}{l}\text { Posición de Equivalencia por Defecto (PED): Parcial } \\
\text { La mazamorra es una comida criolla hecha con maíz blanco partido y hervido, que se come fría o caliente, con o sin leche y a veces } \\
\text { con azúcar, miel o panela. La generalización 'crumbs', que significa migajas, rescata la intención del TO que describe una situación de } \\
\text { miseria, sin embargo causa modulación por cuanto recurre a otro tipo de alimento. }\end{array}$ \\
\hline
\end{tabular}

\begin{tabular}{|l|l|c|}
\hline P. & \multicolumn{1}{|c|}{ CULTUREMA TO } & CATEGORIA GRAMATICAL \\
\hline 96 & $\begin{array}{l}\text { Tenía una medallita de la Virgen de los Remedios colgada en el cuello (...), y en la } \\
\text { muñeca derecha la apretada esclava de cobre de los niños-en-cruz. }\end{array}$ & $\begin{array}{l}\text { Sustantivos con artículos y } \\
\text { preposición }\end{array}$ \\
\hline P. CULTUREMA TM & \multicolumn{1}{|c|}{$\begin{array}{l}\text { TéCNICA DE TRADUCCIÓN } \\
\text { was the tight copper bracelet of the niños-en-cruz amulet. }\end{array}$} & Préstamo puro con descripción \\
\hline $\begin{array}{l}\text { Posición de Equivalencia por Defecto (PED):Total } \\
\text { En el primer caso se observa el uso de una adaptación para recrear la advocación mencionada en el TO 'la Virgen de los Remedios', } \\
\text { que consiste en una de las manifestaciones marianas más extendidas en la cultura origen; especialmente en la region Caribe de } \\
\text { Colombia, donde el pueblo de Riohacha conmemora cada año con una gran fiesta un milagro ocurrido en 1663. La adaptación 'Our } \\
\text { Lady of Help' no corresponde a esta advocación, sino a la de Nuestra Señora del Perpetuo Socorro. }\end{array}$ \\
$\begin{array}{l}\text { El segundo caso hace referencia a un amuleto de brujería, traído a Latinoamérica por esclavos africanos. El amuleto puede } \\
\text { representarse de diferentes formas, desde pequeños muñecos hasta tatuajes en forma de cruces sobrepuestas. El TO lo describe } \\
\text { en forma de esclava de cobre. Estos amuletos llamados niños en cruz pueden otorgar una fuerza sobrehumana a su portador, } \\
\text { pueden usarse para tener la habilidad de enamorar mujeres, o para ser el mejor desempeñando un tarea específica. Esta función } \\
\text { del amuleto es explicada en el segundo fragmento donde José Arcadio demuestra una fuerza descomunal y alguien destaca que } \\
\text { porta este amuleto. Por la especificidad del artilugio, se evidencia el uso de un préstamo puro con descripción en la traducción del } \\
\text { primer fragmento (se añadió el sustantivo amulet para ayudar a la comprensión del lector meta). }\end{array}$ \\
\hline
\end{tabular}




\section{Patrimonio cultural}

\begin{tabular}{|l|l|c|}
\hline P. & \multicolumn{1}{|c|}{ CULTUREMA TO } & CATEGORIA GRAMATICAL \\
\hline 335 & $\begin{array}{l}\text { (..) Santa Sofía de la Piedad que no estrenaba un traje desde los tiempos del ruido, } \\
(\ldots)\end{array}$ & Preposición con sustantivos \\
\hline P. & \multicolumn{1}{|c|}{ CULTUREMA TM } & TÉCNICA DE TRADUCCIÓN \\
\hline 364 & $\begin{array}{l}\text { (...) Santa Sofía de la Piedad, who had not had a new dress since the time of all } \\
\text { the noise, (...) }\end{array}$ & Traducción literal \\
\hline $\begin{array}{l}\text { Posición de Equivalencia por Defecto (PED): parcial } \\
\text { El tiempo del Ruido es el nombre que recibió el fenómeno ocurrido el 9 de marzo de 1687 en la entonces villa de Santafé de Bogotá. } \\
\text { Se caracterizó por un ruido misterioso y fortísimo de origen desconocido, acompañado de un intenso olor a azufre, con una duración } \\
\text { aproximada de } 15 \text { minutos, y generó pánico colectivo en los habitantes de la ciudad. La expresión «Tiempo del Ruido» se convirtió en } \\
\text { un recurso usual dentro de los habitantes del Virreinato de la Nueva Granada (actual Colombia) para designar algún hecho ocurrido } \\
\text { tiempo atrás. Por estas características la expresión se considera parte del patrimonio cultural del TO y sus características no logran ser } \\
\text { rescatadas mediante la traducción literal. }\end{array}$ \\
\hline \multicolumn{2}{|c|}{} \\
\hline \multicolumn{2}{|c|}{ Rango de Equivalencia (RE): (...) Santa Sofía de la Piedad, who had not had a new dress in a million years, (...) } \\
\hline
\end{tabular}

\begin{tabular}{|l|c|c|}
\hline P. & \multicolumn{1}{|c|}{ CULTUREMA TO } & CATEGOR/A GRAMATICAL \\
\hline 340 & Amaneció muerta el jueves santo. & Sustantivo y adjetivo \\
\hline P. & CULTUREMA TM & TÉCNICA DE TRADUCCIÓN \\
\hline 369 & They found her dead on the morning of Good Friday. & Adaptación \\
\hline $\begin{array}{l}\text { Posición de Equivalencia por Defecto (PED): parcial } \\
\text { El TM pretende ubicar al lector en la conmemoración cristiana de la Semana Santa y con este propósito utiliza la adaptación 'Good } \\
\text { Friday' que es el día de la crucifixión y muerte de Jesús y no el jueves santo cuyo equivalente acuñado es `Holy Thursday', pero } \\
\text { quizás menos familiar para el lector de la lengua meta. }\end{array}$ \\
\hline
\end{tabular}

\section{Medio natural}

\begin{tabular}{|l|l|l|}
\hline P. & \multicolumn{1}{|c|}{ CULTUREMA TO } & CATEGORÍA GRAMATICAL \\
\hline 17 & $\begin{array}{l}\text { (...) y pidió el concurso de todos para abrir una trocha que pusiera a Macondo en } \\
\text { contacto con los grandes inventos. }\end{array}$ & Verbo con sustantivo \\
\hline P. & \multicolumn{1}{|c|}{ CULTUREMA TM } & TÉCNICA DE TRADUCCIÓN \\
\hline 10 & $\begin{array}{l}\text { (...) and asked the assembled group to open a way that would put Macondo in contact } \\
\text { with the great inventions. }\end{array}$ & Generalización \\
\hline $\begin{array}{l}\text { Posición de Equivalencia por Defecto (PED): Parcial } \\
\text { Se evidencia la técnica de generalización en way, que describe una vía cualquiera y no una con características propias de la } \\
\text { ruralidad como la trocha. }\end{array}$ \\
\hline $\begin{array}{l}\text { Rango de Equivalencia (RE): } \\
\text { (...) and asked the assembled group to open a footpath / rural path that would put Macondo in contact with the great inventions. }\end{array}$
\end{tabular}

\begin{tabular}{|l|l|c|}
\hline P. & \multicolumn{1}{|c|}{ CULTUREMA TO } & CATEGORIA GRAMATICAL \\
\hline 181 & $\begin{array}{l}\text { El acto se celebró a veinte kilómetros de Macondo, a la sombra de una ceiba } \\
\text { gigantesca (...) }\end{array}$ & Sustantivo con adjetivo \\
\hline P. & \multicolumn{1}{|c|}{ CULTUREMA TM } & TÉCNICA DE TRADUCCIÓN \\
\hline 191 & $\begin{array}{l}\text { The ceremony took place fifteen miles from Macondo in the shade of a gigantic } \\
\text { ceiba tree (...) }\end{array}$ & Amplificación \\
\hline $\begin{array}{l}\text { Posición de Equivalencia por Defecto (PED): } \\
\text { De la misma forma que en el culturema anterior, el traductor agrega información para ayudar al lector meta a comprender, en este } \\
\text { caso, que la ceiba es un árbol. }\end{array}$ \\
\hline
\end{tabular}




\section{Resultados y discusión}

En este apartado presentamos los resultados de la investigación en términos cuantitativos. En la primera columna aparece el listado de técnicas identificadas (17 de 18. No se evidenció sustitución), y en las siguientes cuatro encontramos los ámbitos culturales. El número entre paréntesis corresponde a la cantidad de culturemas clasificados por ámbito (es importante aclarar que el total, con relación a la técnica, es superior al número en paréntesis porque en algunos casos un culturema fue recreado mediante el uso de más de una técnica). La siguiente columna corresponde a la posición de equivalencia por defecto, subdividida con el propósito de cruzar la técnica de traducción con el tipo de equivalencia (total, parcial y nula) lograda.

Tabla 2. Relación de las técnicas de traducción con los ámbitos culturales y la posición de equivalencia por defecto (PED)

\begin{tabular}{|c|c|c|c|c|c|c|c|}
\hline \multirow{2}{*}{$\begin{array}{c}\text { TÉCNICAS } \\
\text { DE TRADUCCIÓN }\end{array}$} & \multicolumn{4}{|c|}{ ÁMBITOS CULTURALES } & \multicolumn{3}{|c|}{$\begin{array}{l}\text { Posición de Equivalencia por Defecto } \\
\text { (PED) }\end{array}$} \\
\hline & $\begin{array}{c}\text { Cultura } \\
\text { lingüística } \\
(50) \\
\end{array}$ & $\begin{array}{c}\text { Cultura } \\
\text { social } \\
\text { (23) }\end{array}$ & $\begin{array}{c}\text { Patrimonio } \\
\text { cultural } \\
(8)\end{array}$ & $\begin{array}{c}\text { Medio } \\
\text { natural } \\
(5)\end{array}$ & Total & Parcial & Nula \\
\hline Adaptación & 8 & 4 & 2 & 0 & 8 & 5 & 0 \\
\hline Ampliación lingüística & 3 & 0 & 0 & 0 & 3 & 0 & 0 \\
\hline Compensación & 1 & 0 & 0 & 0 & 0 & 2 & 0 \\
\hline Comprensión lingüistica & 3 & 0 & 1 & 0 & 4 & 0 & 0 \\
\hline Creación discursiva & 6 & 0 & 0 & 0 & 2 & 3 & 1 \\
\hline Descripción & 6 & 2 & 1 & 1 & 8 & 2 & 0 \\
\hline Equivalente acuñado & 13 & 4 & 1 & 1 & 16 & 2 & 0 \\
\hline Reducción & 7 & 3 & 1 & 0 & 5 & 6 & 0 \\
\hline Traducción literal & 5 & 2 & 1 & 0 & 5 & 3 & 0 \\
\hline Transposición & 2 & 0 & 0 & 0 & 0 & 1 & 0 \\
\hline Variación & 2 & 0 & 0 & 0 & 0 & 2 & 0 \\
\hline No. Total & 79 & 31 & 11 & 9 & 73 & 49 & 3 \\
\hline
\end{tabular}

Como lo ilustra la Tabla 2, la muestra consistió en 84 culturemas (dos con doble clasificación) recreados mediante 125 técnicas de traducción. El 58.4 \% de las recreaciones se ubican en la posición de equivalencia por defecto total, el $39.2 \%$ en parcial, y el $2.4 \%$ en nula.

Una visión global de los resultados evidencia que la generalización fue la técnica de traducción más utilizada con 22 recreaciones, seguida por el equivalente acuñado con 19 y se destacó la posición de equivalencia por defecto total. 
También es posible verificar la PED que alcanzó cada técnica de traducción. El equivalente acuñado obtuvo 16 casos con equivalencia total y 2 con parcial; por su parte, la generalización fue la segunda técnica más utilizada con 12 recreaciones en PED parcial y 9 con PED total.

\subsection{Discusión}

Queremos destacar, en relación con la posición de equivalencia por defecto, que la mayor parte de los culturemas identificados fueron recreados con tipo de equivalencia total y solo registramos dos casos de equivalencia nula. Lo anterior nos permite inferir que los recursos de generalización y equivalente acuñado logran un grado alto de equivalencia, fiel a la intención del texto original. En cuanto a las otras técnicas, como adaptación, comprensión lingüística, reducción y variación, encontramos que coinciden con el planteamiento de Shveitser (1988) sobre la traducción vista como un juego regido por el principio de "mínimas pérdidas, máximas ganancias", pues todas ellas se mantuvieron dentro de la posición de equivalencia por defecto total y parcial.

Las técnicas de ampliación lingüística, amplificación, descripción y particularización fueron menos frecuentes, pero lograron una posición de equivalencia total en la mayoría de sus apariciones. Es probable que esto se deba a su característica común de brindar información adicional o más concreta, de manera que el lector comprenda fácilmente el sentido original de la expresión, pero, a cambio, le resta fluidez y naturalidad al texto meta. Algunas otras técnicas, como el calco, el préstamo puro y la traducción literal, presentan rasgos de la corriente lingüística y se orientan a preservar las formas estructurales del original. Aunque no logran alcanzar en todos los casos la equivalencia total, su uso le otorga un matiz de exotismo al texto meta y ubica al lector en un espacio y una cultura diferentes.

Encontramos además que las técnicas utilizadas para la recreación de los culturemas corresponden a la premisa que expone Bolaños (2016b), de acuerdo con la cual el principal elemento común entre la traducción con orientación lingüística y la corriente cultural del funcionalismo es el reconocimiento de la traducción como un proceso comunicativo, que busca asegurar la comprensión del original por parte del receptor en la cultura meta.

\section{Conclusiones}

Este análisis nos permitió experimentar y encontrar útil la integración de dos corrientes traductológicas como la lingüística y la cultural de manera funcional para 
estudiar tanto el procedimiento como el resultado de una traducción. Comprobamos, además, que el culturema es en efecto una especificidad patente que solo es descubierta en contraste con otra realidad y cuyos aspectos análogos no están definidos de antemano porque dependen siempre del contexto, tal como lo advirtió Molina (2001).

Esto nos lleva a definir el culturema como un punto de encuentro entre las diferencias que constituyen la identidad de los pueblos y cuyo contraste permite el descubrimiento de esa compleja construcción llamada cultura. Creemos, por lo tanto, que esto solo es posible cuando corremos el riesgo de asomarnos al mundo del otro con atenta curiosidad.

En cuanto a las prioridades que guiaron esta traducción literaria, pudimos identificar la fidelidad como el criterio principal usado para llevar al mundo anglófono la voz de uno de los autores más reconocidos del boom latinoamericano en la década del 70. La permanencia de la intención original se evidenció en el alto porcentaje de equivalencias totales, porque, en palabras de Rabassa (2005), "la mejor traducción es la más cercana y no la que pretende ser idéntica"1 (p. 32).

Por último, queremos esbozar algunos caminos investigativos que pueden surgir de esta propuesta y del instrumento aquí planteado, pues consideramos fundamental su replicación para descubrir nuevas posibilidades y limitaciones. El diseño es flexible y se presta para analizar no solo un corpus doble, sino múltiple, y el rango de equivalencia deja siempre la puerta abierta para nuevas propuestas de traducción. Según el enfoque y el interés del investigador, este instrumento es útil también para desvelar los criterios en una traducción e incluso para describir el estilo predominante del traductor. Otras posibilidades son estudios sincrónicos y diacrónicos para verificar cómo han cambiado las normas de traducción en un periodo determinado de la historia. También sería posible contrastar diferentes traducciones de una obra destinadas a un mismo público meta, para detectar el tipo de instrucciones o intereses editoriales (que usualmente responden a las características culturales, sociales y políticas de la lengua meta).

Atendiendo a los principios dinámicos que rigieron este trabajo, esperamos que el instrumento propuesto sea implementado, modificado, refutado y pueda, en definitiva, ser útil para comprender un poco mejor la noción de culturema como elemento revelador de diferencias que nos hacen únicos, pero que nos permiten reencontrarnos.

1 Traducción libre de la autora. 


\section{Referencias}

Agar, M. (2006). Culture: Can you take it anywhere? Invited lecture presented at the Gevirtz Graduate School of Education, University of California at Santa Barbara. International Journal of Qualitative Methods, 5(2), 1-16.

Bolaños Cuellar, S. (2008). Towards an Intergrated Translation Approach: Proposal of Dynamic Translation Model (Doctoral dissertation, Ph. D. Dissertation. Hamburg: Hamburg University).

Bolaños Cuéllar, S. (2016a). Equivalence within the dynamic translation model (DTM): Default equivalence position, equivalence range, initiator's instructions, and translational norms. Forma y Función, 29(2), 183-201.

Bolaños Cuéllar, S. (2016b). Introducción a la traductología: autores, textos y comentarios. Editorial Universidad del Rosario.

i Cortés, C. (1999). Ovidi. Traducción y cultura, de la ideología al texto. Salamanca: Ediciones Colegio de España.

García Márquez, G. (2012). Cien años de soledad. Bogotá: Norma.

Marquez, G. G. (1970). One Hundred Years of Solitude, trans. Gregory Rabassa. New York: Harper \& Row, 1, 18.

Henneke, A. (2015). Traducción y cultura: reflexiones sobre la dimensión cultural de textos y su importancia para la traducción. Cuadernos de Lingüística Hispánica, (26), 103119.

Hatim, B., \& Mason, I. (1995). Teoría de la traducción: una aproximación al discurso. Grupo Planeta (GBS).

Molina Martínez, L. (2001). Análisis descriptivo de la traducción de los culturemas árabeespañol (Doctoral dissertation, Universitat Autònoma de Barcelona).

Molina, L., \& Hurtado Albir, A. (2002). Translation techniques revisited: A dynamic and functionalist approach. Meta: Journal des Traducteurs/Meta: Translators' Journal, $47(4), 498-512$.

Nida, E. A., \& Taber, C. R. (1974). The Theory and Practice of Translation Leiden. Netherlands: The United Bible Societies.

Nord, C. (1997). Translation theories explained: translating as a purposeful activity. Manchester: St. Jerome.

Rabassa, G. (2005). If this be treason: translation and its dyscontents: a memoir. New Directions Publishing. 